\title{
Long-term Survival of a Patient with a Large Hepatocellular Carcinoma with Main Portal Vein Tumor Thrombosis and Spontaneous Tumor Rupture
}

\author{
Hyung-Woo Lee, Gi-Ae Kim, Chi Hyuk Oh, Jae-Jun Shim, Byung-Ho Kim \\ Department of Internal Medicine, Kyung Hee University School of Medicine, Seoul, Korea
}

Received Dec. 27, 2019

Revised Feb. 28, 2020

Accepted Mar. 1, 2020
Optimal treatments for patients with advanced hepatocellular carcinoma (HCC) are still limited and their prognosis remains dismal. Yet, there have been rare cases that have shed light on longer survival in these patients assisted by various treatments. This paper aims to present an extraordinary case of far advanced HCC that had been properly managed in spite of continuous recurrence. A patient visited the hospital with a ruptured large HCC with main portal vein tumor thrombosis but survived longer than 14 years owing to active and prompt interventions. ( $\mathrm{J}$ Liver Cancer 2020;20:148-153)

Keywords: Carcinoma; Hepatocellular; Chemoembolization; Therapeutic; Long-term care

\section{INTRODUCTION}

Liver cancer is a major health problem in the Republic of Korea and is the second major cause of cancer-related death. ${ }^{1}$ Although systemic therapy for patients with advanced hepatocellular carcinoma (HCC) has recently improved with the introduction of new molecularly targeted agents and immunotherapies, prognosis of those patients has not been satisfactory. ${ }^{2,3}$ Meanwhile, diverse treatment approaches involving a combination of various local therapies and systemic treatments have been administered in a sequential or concurrent manner. ${ }^{4-6}$

We would like to report an extraordinary case of a patient who presented with a large HCC with main portal vein tumor thrombosis (PVTT) and showed long-term survival in

\footnotetext{
Corresponding author : Jae-Jun Shim

Department of Internal Medicine, Kyung Hee University School of Medicine, 23 Kyungheedae-ro, Dongdaemun-gu, Seoul 02447, Korea Tel. +82-2-958-8155, Fax. +82-2-968-1848

E-mail; joyshim@khu.ac.kr

https://orcid.org/0000-0003-2497-8663
}

spite of continuous recurrence. Moreover, the tumor was already ruptured at the time of presentation and the patient was hemodynamically unstable due to intraperitoneal massive bleeding. However, after over 14 years, he is still alive and currently cancer-free following eight sessions of transarterial chemoembolization (TACE), two tumor resections, six cycles of systemic chemotherapy, and one session of radiotherapy. This study was approved by the Institutional Review Board of Kyung Hee University (KHSIRB 2020-02-036).

\section{CASE REPORT}

\section{Clinical findings}

A 55-year old man visited the hospital due to severe abdominal pain for 1 day. He had been healthy without any medical history. He had been drinking a bottle of makgeolli (traditional raw rice wine, about $50 \mathrm{~g}$ of alcohol in a bottle) daily and smoked 40-packs over the years. On physical examination, his abdomen was flat but rigid. His initial blood pressure was $70 / 50 \mathrm{mmHg}$ and heart rate was 133 beats per 
minute. Initial hemoglobin level was $13.7 \mathrm{~g} / \mathrm{dL}$, which had subsequently dropped rapidly to $9.1 \mathrm{~g} / \mathrm{dL}$. Plasma levels of alanine aminotransferase (ALT) and aspartate aminotransferase (AST) were 57 IU/L and $50 \mathrm{IU} / \mathrm{L}$, respectively. The results of the other blood tests were within normal ranges. Serology tests revealed positive for hepatitis B surface antigen, negative for hepatitis $\mathrm{B}$ envelope antigen, positive for hepatitis B surface antibody, positive for hepatitis B envelope antibody, and negative for hepatitis $\mathrm{C}$ antibody. The Child-Pugh score was 6 points (grade A) and Model for End-Stage Liver Disease score was 7 . His serum alpha-fetoprotein level was 2,179 ng/mL. However, his initial serum HBV-DNA level was not available on admission. As mentioned earlier, serum AST and ALT were not more than twice the normal level (80 IU/L). Therefore, antiviral treatment was not initiated following the guideline for chronic hepatitis B treatment at the time.

\section{Image findings}

An initial abdominal computed tomography (CT) scan showed a hypervascular hepatic mass involving the left lobe and caudate lobe $(16.5 \mathrm{~cm})$. Tumor thrombosis was also noted in the left and main portal veins. A large amount of bloody fluid had accumulated in the peritoneal cavity (Fig. 1).

\section{Diagnosis and treatment}

\section{1) Initial treatment and outcome}

The patient was stabilized with transfusion and urgent TACE (\#1). However, a viable tumor was found in a followup CT scan 1 month later. Tumor thrombosis remained in the left portal and proximal tip of the main portal vein. Chest CT scan and positron emission tomography-computed tomography (PET-CT) showed no extrahepatic metastasis. Since the patient had good liver function, the left lobe and caudate lobe were surgically resected with portal vein thrombectomy at 1 month after initial diagnosis. On histologic examination, the resection margin was clear. The resected liver parenchyma showed proliferation of the bile duct and fibrosis with chronic inflammatory cell infiltration. However, microscopic vascular and lymphatic tumor invasion was reported. After successful resection of the tumor in the left lobe, two sessions of TACE (\#2, \#3) were performed subsequently for a small HCC-like lesion in the right lobe (2 and 8 months, respectively).

\section{2) The first recurrence}

The patient showed no visible recurrent tumors in the liver until two metastatic nodules were found in the lung at 17 months of initial diagnosis. On December 2006, a PET-CT scan showed a metastatic lung nodule $(1 \mathrm{~cm})$ at the left upper lobe and hilar mass $(2.5 \mathrm{~cm})$ in the left mediastinum (Fig. 2). The lesion was confirmed as metastatic HCC by biopsy. From January to May 2007 (18-22 months), six cycles
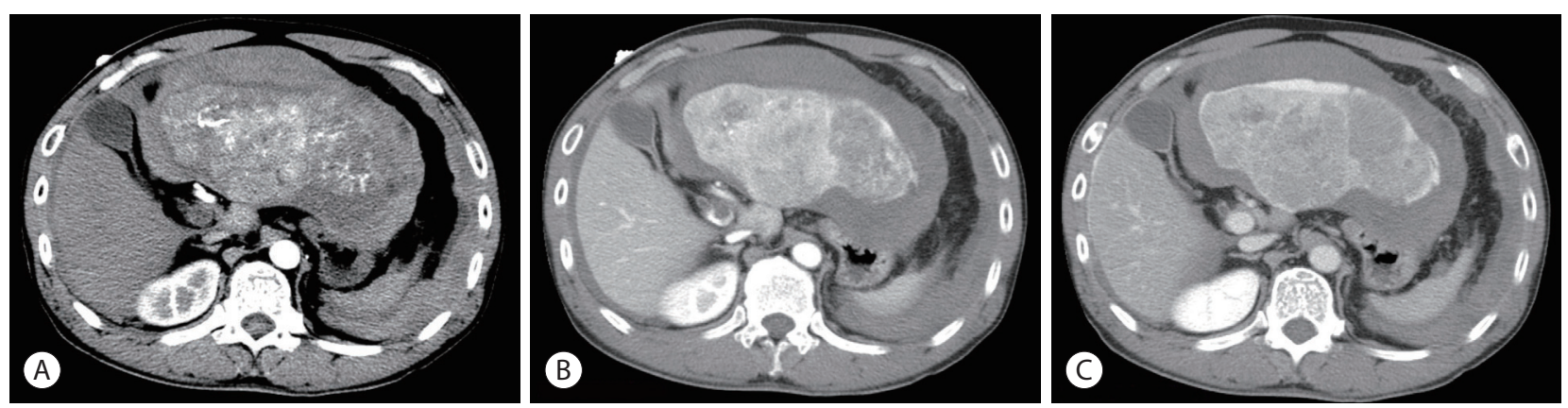

Figure 1. Initial abdomen computed tomography images. (A) arterial phase, (B) portal phase and (C) delayed phase, showing a huge liver mass in the left lobe with hemoperitoneum due to hepatic tumor rupture, measuring $16.5 \mathrm{~cm}$ and thrombus extending from the distal main portal to the left portal vein. 

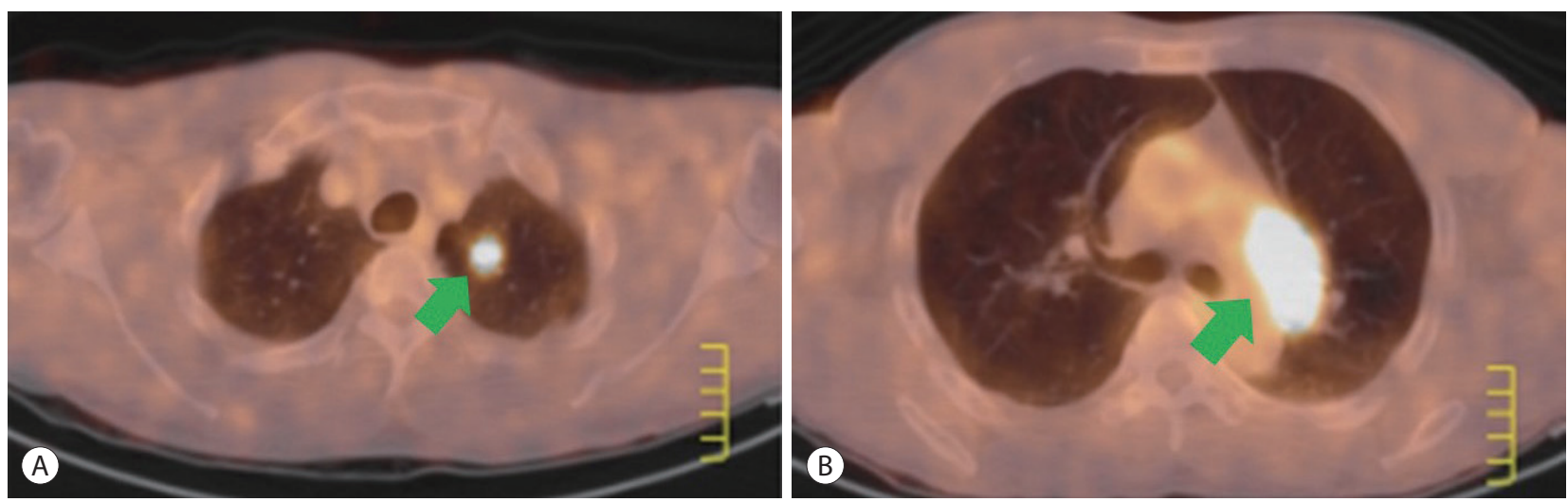

Figure 2. Positron emission tomography-computed tomography scan showing recurrent metastatic hepatocellular carcinomas at the left upper lobe of the lung $(A$, arrow) and para aortic lymph node $(B$, arrow) at 16 months following surgical resection.

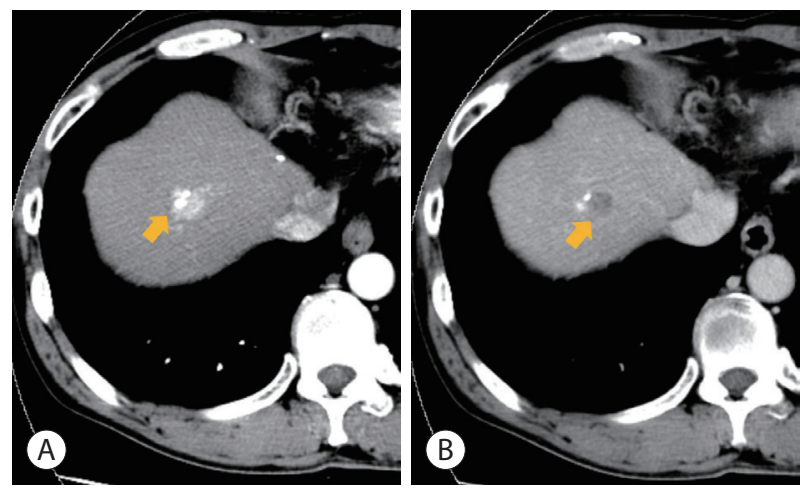

Figure 3. A recurrent hepatocellular carcinoma at segment 8 following repeated transcatheter arterial chemoembolization (fifth and sixth). (A) Arterial phase (arrow), (B) delayed phase (arrow).

of systemic chemotherapy every 3 weeks were performed. One cycle of chemotherapy consisted of fluorouracil (5-FU) $500 \mathrm{mg} / \mathrm{m}^{2}$, doxorubicin $30 \mathrm{mg} / \mathrm{m}^{2}$, cisplatin $60 \mathrm{mg} / \mathrm{m}^{2}$ on day 1 and 5 -FU $500 \mathrm{mg} / \mathrm{m}^{2}$ on days 2 and 3. All metastatic tumors after systemic chemotherapy disappeared on followup imaging. No extrahepatic metastatic tumors were found during the subsequent follow-up period.

\section{3) The second and third recurrence}

On October 2007 (27 months), intrahepatic recurrence of HCC was confirmed by periodic CT scans. A recurrent tumor measuring $1 \mathrm{~cm}$ was observed in segment 8 . The tumor was treated with TACE (\#4) successfully. The third recurrence occurred near the previously recurred tumor at segment 8 . The tumor was $1.7 \mathrm{~cm}$ in size and showed a typical enhancement pattern in a dynamic CT scan. Two sessions of TACE (\#5, \#6) were performed at 38 and 41 months after initial diagnosis, respectively. However, the tumor increased despite treatments (Fig. 3). The serum AST and ALT levels at the time of surgery were $204 \mathrm{IU} / \mathrm{L}$ and $269 \mathrm{IU} / \mathrm{L}$, respectively. The Child-Pugh score was 6. The recurred tumor was surgically resected in August 2009 (49 months later). The resected hepatic parenchyma showed liver cirrhosis.

\section{4) The fourth recurrence}

Fifteen months after the second resection, the fourth tumor recurrence occurred near the resection margin. Liver magnetic resonance imaging (MRI) scan confirmed a recurrent small HCC measuring $0.8 \mathrm{~cm}$ on segment 8 . Initially, radiofrequency ablation was considered since it is a curative treatment; however, the lesion was too small to be detected on the ultrasonography. Alternatively, radiotherapy (RT) was considered and at the time of RT treatment, the serum AST and ALT levels were $65 \mathrm{IU} / \mathrm{L}$ and $115 \mathrm{IU} / \mathrm{L}$, respectively. A total of 4,500 cGy was administered with a daily dose of $300 \mathrm{cGy}$. There was no further recurrence around the tumor. Following radiotherapy, reactivation of hepatitis B virus (HBV) occurred. Serum ALT was $652 \mathrm{U} / \mathrm{L}$ and HBV DNA level was $667,781 \mathrm{IU} / \mathrm{mL}$. Entecavir $(0.5 \mathrm{mg})$ was initiated and ALT normalized in 6 months. Serum HBV DNA was undetectable in 12 months. 

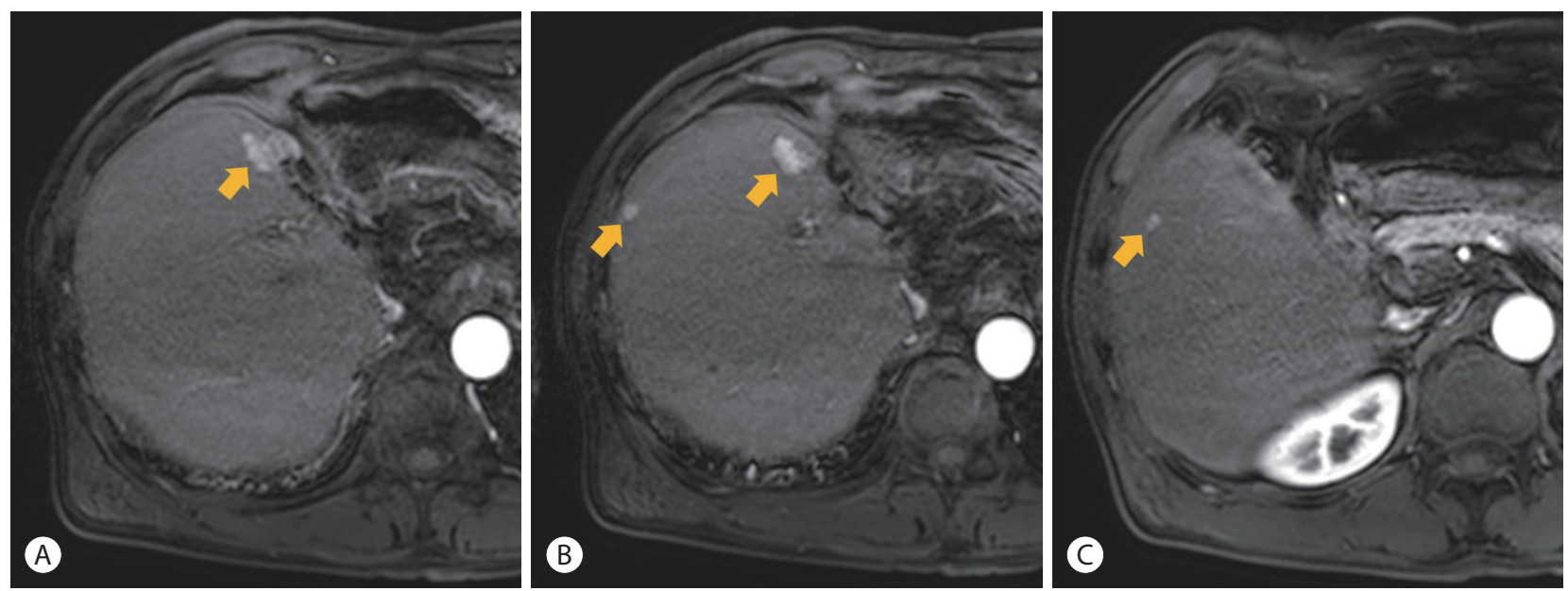

Figure 4. Liver magnetic resonance imaging images showing recurrent hepatocellular carcinomas (arrow) in an arterial phase. After 8 years of the first hepatic resection, multiple tumors recurred at segment 5 and 8 .

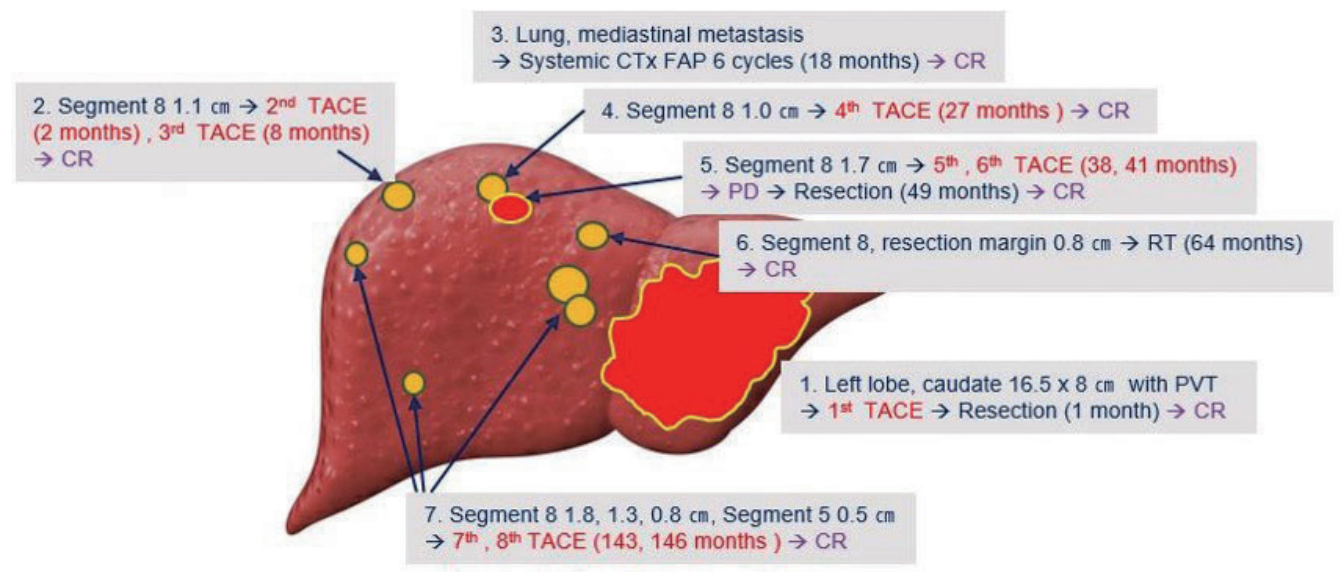

Figure 5. Case summary showing tumor location and treatments for 15 years. $\mathrm{TACE}$, transcatheter arterial chemoembolization; $\mathrm{CR}$, complete remission; CTx, chemotherapy; FAP, fluorouracil, adriamycin, and cisplatin; PD, progressive disease; RT, radiotherapy; PVT, portal vein thrombosis.

\section{5) The fifth recurrence}

Following RT, liver CT or MRI was conducted every several months. For 6.5 years, there was no more recurrence. In June 2017, liver MRI showed the 5th recurrence in the liver. Three recurrent tumors were observed in segment 8 . A nodular lesion measuring $1.8 \mathrm{~cm}$ was found in the anterior border of the resection margin (Fig. 4A). Another nodule measuring $0.8 \mathrm{~cm}$ was found at the lateral subcapsular portion of segment 8 (Fig. 4B). Another one measuring $0.5 \mathrm{~cm}$ was found at segment 5 (Fig. 4C). These nodular lesions showed a typically enhancing pattern and were confirmed to be recurrent HCCs even though the two lesions were less than $1.0 \mathrm{~cm}$ in size. These lesions were treated with two sessions of
TACE $(\# 7, \# 8)$ at 143 and 146 months after initial diagnosis, respectively. Imaging tests following TACE showed no viable portion remained around the lesions until July 2019 (168 months). The liver function of the patient was persistently stable with Child-Pugh A during follow-up.

In summary, a large ruptured HCC with portal vein thrombosis in the left lobe was resected following TACE. Extrahepatic recurrence in the lung and mediastinum were all treated with systemic chemotherapy. During the 13 years after systemic treatment, four more intrahepatic recurrences occurred and they were all treated with repeated TACE, RT, or wedge resection (Fig. 5). The patient is still alive with no remnant or recurrent tumors in the liver. 


\section{DISCUSSION}

This case demonstrated that long-term survival is possible in a patient with a large ruptured HCC and main PVTT. A major predictor for good prognosis in the patient might have been a well-preserved liver function and control of underlying liver diseases (HBV and alcohol). Another prognostic factor in the patient might have been sufficient tumor control by two kinds of initial aggressive treatments, surgical resection and systemic chemotherapy. Although the risk of early recurrence was very high, surgery was performed to remove the primary lesions in the liver.

Although the incidence of massive bleeding from a ruptured tumor is decreasing, it is still a life-threatening complication in patients with large HCC. ${ }^{7}$ The prognosis of patients with ruptured HCC is poorer than those with non-ruptured HCC. However, the impact of tumor rupture on the overall prognosis or tumor recurrence was not as strong as other parameters such as, tumor size or underlying liver function. ${ }^{7,8}$

Current guidelines recommend systemic therapy as an initial treatment in patients with advanced HCC. However, in patients with locally advanced HCC and PVTT, the optimal treatment is still controversial. According to a large retrospective study from Japan, resection showed better survival than non-surgical treatments in patients with PVTT limited in the first-order branch. ${ }^{9}$ In patients with localized HCC and type I PVTT, resection showed longer survival than TACE or sorafenib. ${ }^{10}$ Based on these studies, recent Korean guidelines suggest that hepatic resection can be considered in patients with three or fewer intrahepatic tumors with invasion to the hepatic vein, portal vein or bile duct invasion if hepatic function is well preserved and the main portal trunk is not invaded. ${ }^{11}$ The present case does not qualify for surgical resection under the current standard as the tumor had type III PVTT (invasion of main portal vein). Moreover, sorafenib was not available before 2008. However, a couple of benefits were surely expected from an active treatment such as surgical resection since the patient showed relatively well preserved liver function with a good performance status.

The patient eventually showed early extrahepatic metastasis following aggressive surgical resection, which was well an- ticipated given the patient's initial state. As mentioned earlier, conventional chemotherapeutic agents (fluorouracil, doxorubicin, cisplatin) showed very good tumor response in the patient.

Moreover, recurrent tumors in the liver also seemed to be very sensitive to the same chemotherapeutic agent (adriamycin) used in TACE. Despite 5 instances of recurrence, the lesion was found relatively early since follow-up imaging was conducted every several months with liver CT or MRI. In this case, all the small recurrent HCCs were completely treated with one or two sessions of TACE except for the third recurrent tumor. Sensitivity to a chemotherapeutic agent might be associated with better tumor control by TACE in the present case.

Additionally, even though systemic therapy is now considered as a standard of care in patients with HCC and extrahepatic metastasis, recent retrospective studies suggest that a combination with local and systemic therapy might have more beneficial outcomes in terms of better tumor control and survival. ${ }^{12-14}$ Systemic treatment following aggressive control of the primary tumor might provide a higher chance for cure than systemic treatment alone. More well-designed prospective studies are required to reveal its superiority.

The fact that we would like to emphasize once again is that the patient preserved his liver function for a very long period. This enabled many aggressive and continuous treatments for a long time. Furthermore, complete abstinence from alcohol drinking and maintenance of antiviral therapy played an important role. This case clearly highlights the importance of treating underlying liver diseases well, not just tumors.

Finally, it should be noted that the number of TACE sessions performed on the same tumor might impact local tumor control and prognosis. We performed 8 sessions of TACE for the primary and recurrent tumors. We did not perform more than two sessions of TACE for the same tumor. When a sequential treatment showed insufficient tumor response, we immediately switched to surgical resection. The recent Korean clinical practice guidelines recommend a change of treatment when patients show signs of TACE refractory such as, absence of objective response (complete response or partial response), a new vascular invasion, or ex- 
trahepatic metastasis after two consecutive TACE sessions within six months. The definition of TACE failure or refractory differs based on the practice guidelines commonly available in Europe or Japan. Thus, there is no established guideline for properly handling TACE failure or refractory. Moreover, it has been reported that patients who received more than two TACE sessions within 6 months showed an increased chance of disease progression and higher mortality than those who did not. ${ }^{15}$ When patients show TACE refractory, various treatment options can be considered depending on the status of the patient. Systemic treatment can be considered in the case of advanced HCC and hepatic resection, or liver transplantation can be considered when the performance status of a patient is good and surgery is an option. ${ }^{16}$

This case highlights that surgical resection can be a treatment option even in patients with far advanced HCC with portal vein thrombosis. Even though the recurrence rate was very high, recurrent tumors could be detected at an early stage with regular surveillance and can also be successfully controlled with various local therapies. Although the findings of our case cannot be applied to all patients, our case emphasizes the importance of aggressive tumor control for primary and recurrent tumors with various local or systemic treatments even in an advanced tumor stage.

\section{Conflicts of Interest}

The authors have no conflicts to disclose.

\section{REFERENCES}

1. Jung KW, Won YJ, Kong HJ, Lee ES. Cancer statistics in Korea: incidence, mortality, survival, and prevalence in 2016. Cancer Res Treat 2019;51:417-430.

2. Llovet JM, Ricci S, Mazzaferro V, Hilgard P, Gane E, Blanc JF, et al. Sorafenib in advanced hepatocellular carcinoma. N Engl J Med 2008;359:378-390.

3. Kudo M, Finn RS, Qin S, Han KH, Ikeda K, Piscaglia F, et al. Lenvatinib versus sorafenib in first-line treatment of patients with unresectable hepatocellular carcinoma: a randomised phase 3 noninferiority trial. Lancet 2018;391:1163-1173.

4. Yoon SM, Ryoo BY, Lee SJ, Kim JH, Shin JH, An JH, et al. Efficacy and safety of transarterial chemoembolization plus external beam radiotherapy vs sorafenib in hepatocellular carcinoma with macro- scopic vascular invasion: a randomized clinical trial. JAMA Oncol 2018;4:661-669.

5. He M, Li Q, Zou R, Shen J, Fang W, Tan G, et al. Sorafenib plus hepatic arterial infusion of oxaliplatin, fluorouracil, and leucovorin vs sorafenib alone for hepatocellular carcinoma with portal vein invasion: a randomized clinical trial. JAMA Oncol 2019;5:953-960.

6. Fernandes ESM, Rodrigues PD, Álvares-da-Silva MR, Scaffaro LA, Farenzena M, Teixeira UF, et al. Treatment strategies for locally advanced hepatocellular carcinoma. Transl Gastroenterol Hepatol 2019;4:12.

7. Yoshida H, Mamada Y, Taniai N, Uchida E. Spontaneous ruptured hepatocellular carcinoma. Hepatol Res 2016;46:13-21.

8. Aoki T, Kokudo N, Matsuyama Y, Izumi N, Ichida T, Kudo M, et al. Prognostic impact of spontaneous tumor rupture in patients with hepatocellular carcinoma: an analysis of 1160 cases from a nationwide survey. Ann Surg 2014;259:532-542.

9. Kokudo T, Hasegawa K, Matsuyama Y, Takayama T, Izumi N, Kadoya $M$, et al. Survival benefit of liver resection for hepatocellular carcinoma associated with portal vein invasion. J Hepatol 2016;65:938-943.

10. Lee JM, Jang BK, Lee YJ, Choi WY, Choi SM, Chung WJ, et al. Survival outcomes of hepatic resection compared with transarterial chemoembolization or sorafenib for hepatocellular carcinoma with portal vein tumor thrombosis. Clin Mol Hepatol 2016;22:160-167.

11. Korean Liver Cancer Association; National Cancer Center. 2018 Korean Liver Cancer Association-National Cancer Center Korea practice guideline for the management of hepatocellular carcinoma. Gut Liver 2019;13:227-299.

12. Li L, Zhao W, Wang M, Hu J, Wang E, Zhao Y, et al. Transarterial chemoembolization plus sorafenib for the management of unresectable hepatocellular carcinoma: a systematic review and metaanalysis. BMC Gastroenterol 2018;18:138.

13. Choi GH, Shim JH, Kim MJ, Ryu MH, Ryoo BY, Kang YK, et al. Sorafenib alone versus sorafenib combined with transarterial chemoembolization for advanced-stage hepatocellular carcinoma: results of propensity score analyses. Radiology 2013;269:603-611.

14. Chen J, Lu S, Zhang Y, Xu L, Chen J, Wang J, et al. Sorafenib monotherapy versus sorafenib combined with regional therapies for hepatocellular carcinoma patients with pulmonary oligometastases: a propensity score-matched analysis. J Cancer 2018;9:17451753.

15. Jeon MY, Kim HS, Lim TS, Han DH, Kim BK, Park JY, et al. Refractoriness to transarterial chemoembolization in patients with recurrent hepatocellular carcinoma after curative resection. PLoS One 2019;14:e0214613.

16. Galle PR, Tovoli F, Foerster F, Wörns MA, Cucchetti A, Bolondi L. The treatment of intermediate stage tumours beyond TACE: From surgery to systemic therapy. J Hepatol 2017;67:173-183. 